\title{
Localization of Sound Sensor Wireless Network with a Mobile Beacon
}

\author{
Qinli An \\ Department of Basic Sciences, Air Force Engineering University, \\ $710051 X i$ 'an, China \\ anqinli@126.com
}

\begin{abstract}
Most of wireless sensor network (WSN) applications need the sensor nodes aware of their own locations. In this paper, we adopt a mobile beacon node to construct sensor node localization scheme in a randomly deployed wireless sensor network (WSN). Supposing that each sensor node have one omnidirectional antenna and one microphone, the mobile beacon node fits with two directional antennas and two directional sound sources orienting to the horizontal axis and the inverse respectively. In the proposed scheme, we adopt a multi-modal approach combining radio and sound. The mobile beacon node determines its coordinate via GPS, broadcasts time labels and its coordinate information, and emits sound pulse at the same time as it travels through the WSN. The sensor nodes detect these beacon messages and sound pulses, measure the time delays between radio and sound pulse, then adopt the y-coodinate of the beacon message of the minimum time delay between radio and sound pulse as its own and obtain the $x$-coodinate according to the beacon node antennas 'ID. Our approach can accurately estimate the sensors" locations. It is displayed that the localization scheme, designated as DAS, is robust to various parameters of the WSN and immune to obstacle surroundings through error analysis and computer simulation.
\end{abstract}

Keywords: wireless sensor network (WSN); mobile beacon; beacon node; directional antenna; directional sound source

\section{Introduction}

With the rapid development of wireless sensor network (WSN) technology, many research domains begin to pay close attention to it and put forward a large number of problems to solve. Wireless sensor nodes are usually stationary (static) and are powered by batteries of restricted capacity [1]. There are a large amount of studies about WSNs, e.g., addressing, coverage, data aggregation, deployment, top control, localization, routing, tracking etc. The information collected by sensor nodes and the location of the sensor nodes need to be correlated with for an exact observation of the monitored field. Furthermore, tracking targets needs a lot of location information of the near sensor nodes [2]. Except for these applications, the solutions proposed in many research fields need to know the locations of the sensor nodes. For instance, it is necessary for locations of neighbor sensor nodes to check for the degree of a local coverage [3]. Furthermore, many forwarding and routing schemes need to know the location information of the local sensor nodes [4].

It is easy to think of the global positioning system (GPS) [5] to locate sensor nodes for its prominent precision. However, it is unimaginable cost and energy consumption to fit each sensor node with a GPS receiver in a large scale WSN for its restricted capacity battery. In that case, many cost-effective and energy-efficient localization schemes have

Received (January 25, 2018), Review Result (April 25, 2018), Accepted (May 1, 2018) 
been put forward. The locations of nodes are obtained by multiple pairwise range measurements of senor nodes, those methods is known as Range-based localization schemes. Ranges between nodes are acquired using travel time of signals between nodes, designated as TOA, the signals' arrival time difference between nodes, designated as TDOA, path loss attenuation with range received signal strength indicator, designated as RSSI. The location is determined by the angles between unknown nodes and beacon nodes, designated as AOA [6-18].

There are three main localization schemes of range-based as follows.

Trilateration: Trilateration schemes can be utilized to determine sensor nodes' locations for range measurement between nodes available. As is displayed in Figure 1(a), An unknown node can be localized through range measurements of its three neighbor nodes with available locations.

Triangulation: In the AoA scheme, the unknown node can be localized through the angle measurement from two beacon nodes with available locations which is displayed in Figure 1 (b).

Maximum likelihood multilateration: A precise estimate of location is difficult to obtain for range measurements with noise for the trilateration scheme. Thus, maximum likelihood (ML) estimation schemes are put forward to merge range measurements from multiple neighbor nodes for improving localization precision. In the case, obtaining the locations of the nodes through minimizing the differences between the estimated ranges and the range measurements is displayed in Figure 1(c).

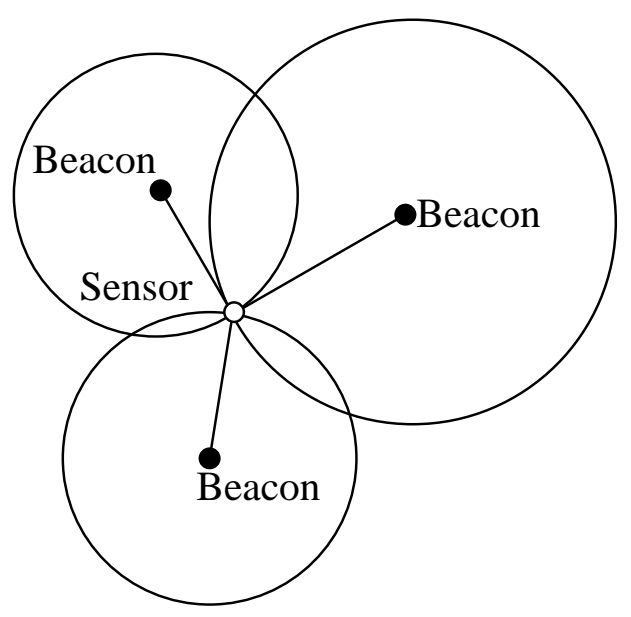

(a) Trilateration

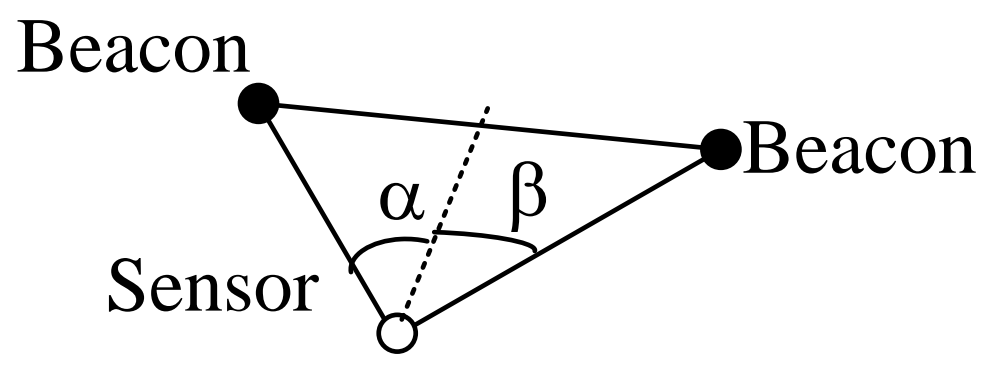

(b) Triangulation 


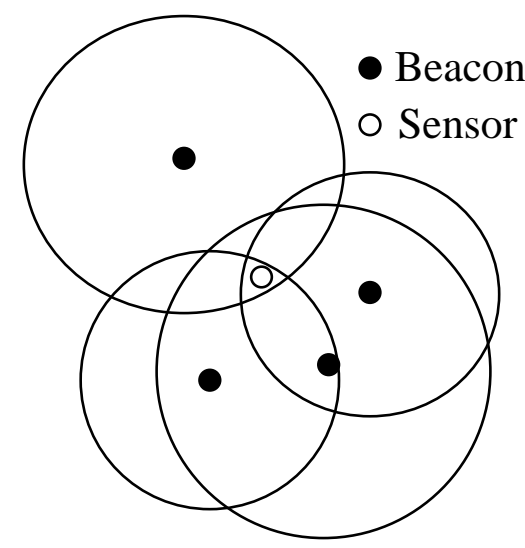

(c) Multilateration

Figure 1. Range-based Localization Schemes

Range-based schemes above have high precision. however, the sensor node cost is enhanced to fit each sensor node with extra hardware for ranging in these schemes.

In a lot part of WSN applications, only rough localization may be enough. So a lot of efficient and cost-effective localization schemes without extra ranging measurements are put forward, designated as range-free localization scheme. In these schemes, the connectivity information between nodes constraints the location of the nodes. The localization of sensor nodes also utilizes beacon nodes for a reference analogical to the range-based schemes $[7,11,12,13,18,19]$. In the section, we give two range-free schemes for instance: convex position estimation scheme [19] and the approximate point-intriangle (APIT) scheme [12].

Convex Position Estimation: As is displayed in Figure 2, The sensor node $\mathrm{S}$ is the neighbour of sensor nodes $\mathrm{A}, \mathrm{B}, \mathrm{C}$ and $\mathrm{D}$ and the sensor nodes $\mathrm{A}, \mathrm{B}, \mathrm{C}$ and $\mathrm{D}$ know their own locations. Asuming that communication range $\mathrm{R}$ of node $\mathrm{A}, \mathrm{B}, \mathrm{C}$ and $\mathrm{D}$ is known, afterwards sensor node $\mathrm{S}$ locate in circles centered at node $\mathrm{A}, \mathrm{B}, \mathrm{C}$ and $\mathrm{D}$ with radius $\mathrm{R}$. As a result, the location of the sensor node $S$ can be obtained through settling an optimisation problem.

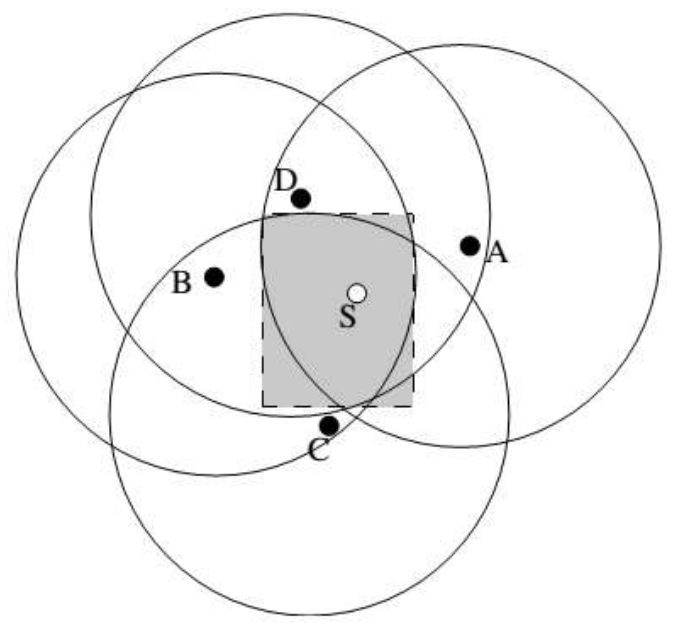

Figure 2. Convex Position Estimation

APIT: In The APIT scheme [12], The RSS information from a large amount of nodes is only utilized to compare received signal strength and not to estimate range between sensor nodes, then estimate the relative locations of the unknown sensor nodes. 


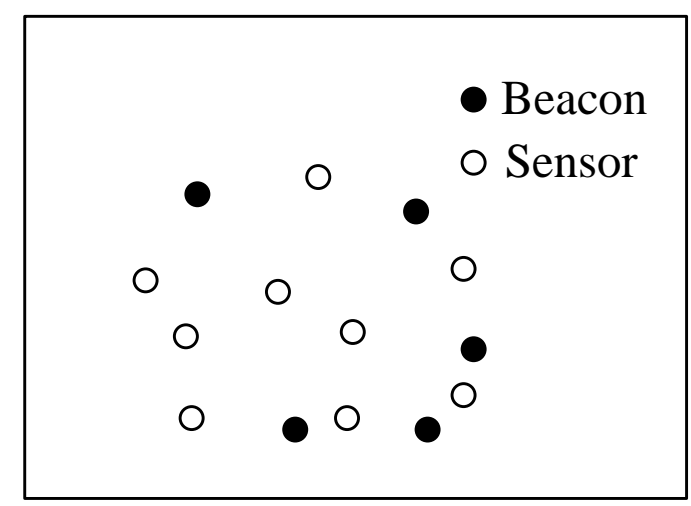

(a) Network Architecture

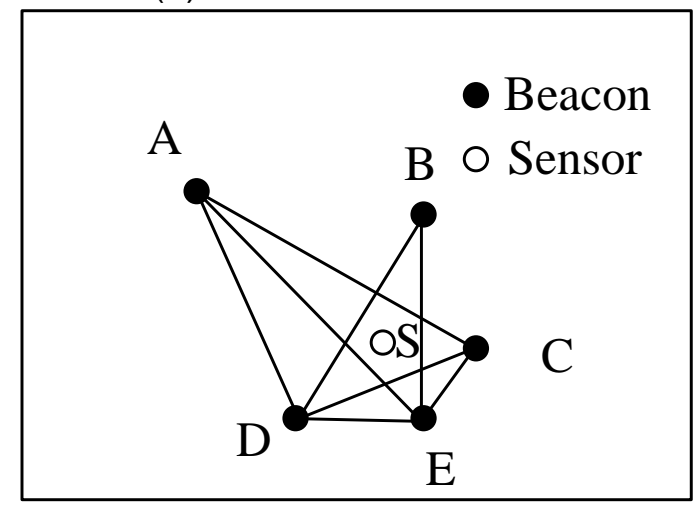

(b) Reference Triangles in the APIT Scheme

Figure 3. The APIT Scheme

As is displayed in Figure 3(a), Assume that the node $\mathrm{S}$ knows the locations of the beacon nodes A, B, C, D, and E. Using any three of the non-collinear beacon nodes, triangles can be constituted as shown in Figure 3(b). Suppose that the sensor node S can judge if it is in or out of these triangles through comparing the received signal strength, then the sensor node can obtain its location using the intersection of these triangles.

Taking into localization scheme account above, the contradiction between the location precision and the cost is unavoidable whether the scheme is range-free or range-based. The localization precision of localization above is partly dependent on sensor node density and topology, furthermore, a lot of sensor node communication is utilized.

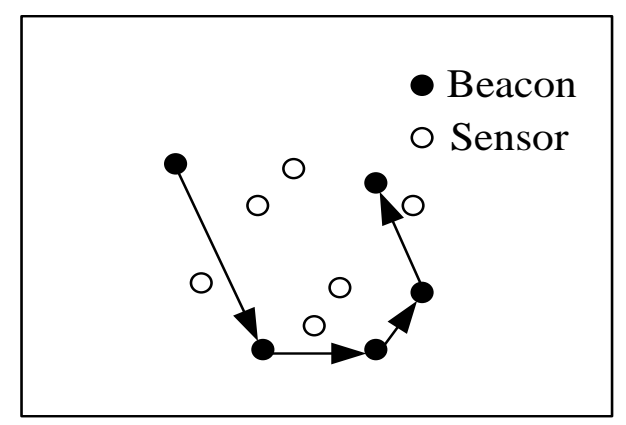

Figure 4. Utilization of Mobile Beacon Node in WSN

Thus the wireless sensor node localization schemes using mobile beacon node are put forward. In the localization schemes, as is displayed in Figure 4, the mobile beacon nodes 
travel through(or fly over) the WSN and issue beacon messages including their present location, time, etc. Then the coordinates of sensor nodes are obtained through range-free or range-based schemes [20-22]. It is ill-famed for omni-directional antenna's radiated energy in entire orientations and giving rise to large localization errors for more surroundings noise. So localization scheme using mobile beacon nodes with directional antennas are proposed [23-24]. These schemes have displayed great practical merits through adopting mobile beacon nodes for sensor node localization.

The localization schemes above only use communication equipment, and sensing equipment isn't used. We consider that a mobile beacon node has communication, beacon, and target functions. Each sensor node determine own location without extra communication and clock synchronization between the other sensor nodes in this scheme.

The sensor localization scheme is implemented as follows. The mobile beacon node is supposed to know its own coordinate (e.g., from GPS), and fitted with directional antennas and directional sound sources. Each sensor node is random deployed in a twodimensional field and supposed to contain a microphone, a signal processor and a transceiver. In the proposed localization scheme, designated as DAS, each mobile beacon node broadcasts beacon message (containing coordinate and time and so on) via two directional antennas meanwhile emits a sound pulse signal via two directional sound sources as it travels through the WSN. Each sensor node computes its own coordinates based on the message received from the mobile beacon nodes and the Time Difference Of Arrival (TDOA) measurements between radio signal and sound pulse signal.

An outline of the paper is as follows. We begin with a model for the directional antenna and sound source. DAS localization scheme are then presented, followed by localization error analysis and its property of obstacle immunization. Computer simulations indicate that the DAS localization scheme are effective, robust to various parameters of WSN and the mobile beacon node, yet immune to obstacle.

\section{Directional Antenna Models and Sound Source Models}

All printed material, including text, illustrations, and charts, must be kept within the parameters of the $815 / 16$-inch (53.75 picas) column length and 5 15/16-inch (36 picas) column width. Please do not write or print outside of the column parameters. Margins are $3.3 \mathrm{~cm}$ on the left side, $3.65 \mathrm{~cm}$ on the right, $2.03 \mathrm{~cm}$ on the top, and $3.05 \mathrm{~cm}$ on the bottom. Paper orientation in all pages should be in portrait style.

As is displayed in Figure 5(a), a main lobe exist in the direction of maximum radiation a directional antenna in accompany with some other side lobes. the side lobes can be suppressed [25] by the side lobe suppression methods. In this paper, the localization scheme ignores these side lobes and approximates the antenna pattern of fan-shaped with a vertex angle of $\theta$ (beam width) as seen in Figure 5(b). The same conclusion is drawn for directional sound sources in similar way. The antenna of beacon node makes use of communication frequencies between $2.41-2.46 \mathrm{GHz}$ and the sound sources central frequency is set up at near $4.4 \mathrm{KHz}$ [26]. 


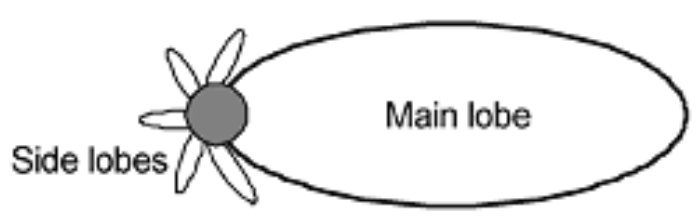

(a)

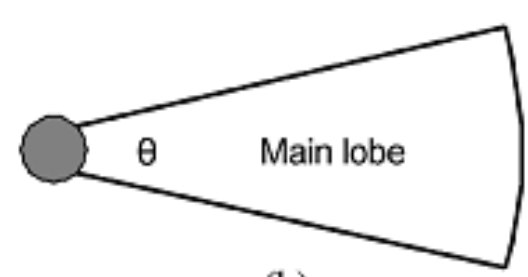

(b)

Figure 5. Directional Antenna and Directional Sound Source Patterns. (a) Real Pattern. (b) Approximate Pattern

\section{DAS Localization Scheme}

As is displayed in Figure 6, the mobile beacon node is fitted with two directional antennas and two directional sound sources with a vertex angle of $\theta$. Furthermore, the mobile beacon nodes is also fitted with a digital compass. The orientation of two directional antennas and two directional sound sources is parallel to the horizontal axis (i.e., the $\mathrm{x}$ axis). The radio and sound range of the main lobe are defined as R. Supposed that the beacon node includes $I D=\{1,2\}$ antennas and each beam $i d$ as displayed in Figure 6. Each sensor node is supposed to be fitted with a microphone and an omnidirectional antenna. The mobile beacon node transmits beacon messages and sound pulses at the same time successively from its $I D$ antennas and sound sources every beacon message delay $T$ second as it travels through the WSN.

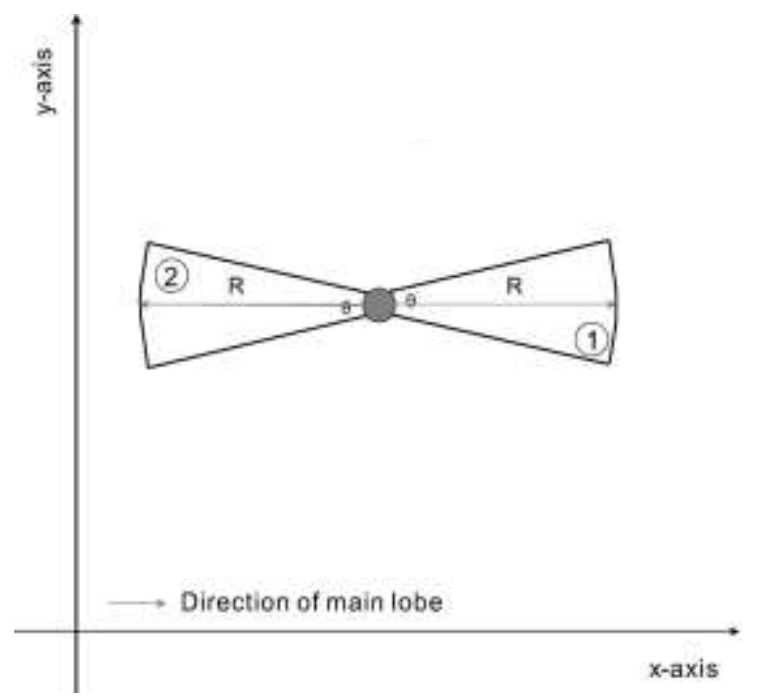

\section{Figure 6. Directional Antenna and Directional Sound Source Patterns of a Mobile Beacon Node}

The beacon message has the form ( $i d$, coordinate, time), where id indicate the antenna index of beacon node which issue beacon message.

The mobile beacon node travels through the WSN by SCAN trajectory [27] along the line, as is displayed in Figure 7. The range between two neighbor line is $2 R$. The choice of $R$ is based on given localization precision. 


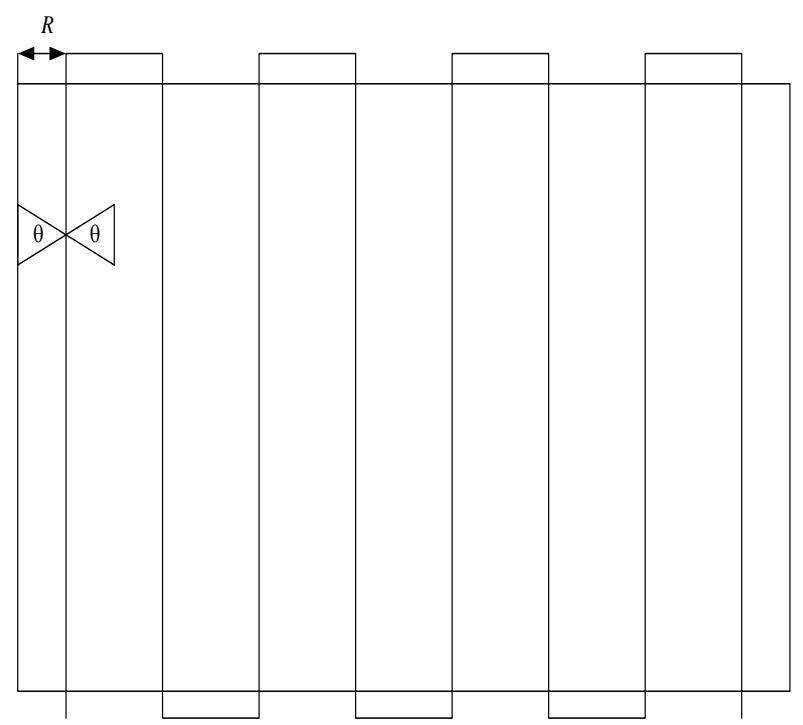

Figure 7. SCAN Trajectory of the Mobile Beacon Node

The beacon node travels through the WSN in a snake-like scheme to locate static sensor node in obstacle surroundings. As is displayed in Figure 8, it exists two obstacles in the WSN, one is rectangle obstacle and the other is triangle Obstacle. Beacon node begins from the left-bottom corner of the WSN to travel through entire sensor field from bottom to top, then right, then from top to bottom, again right and so on [28].

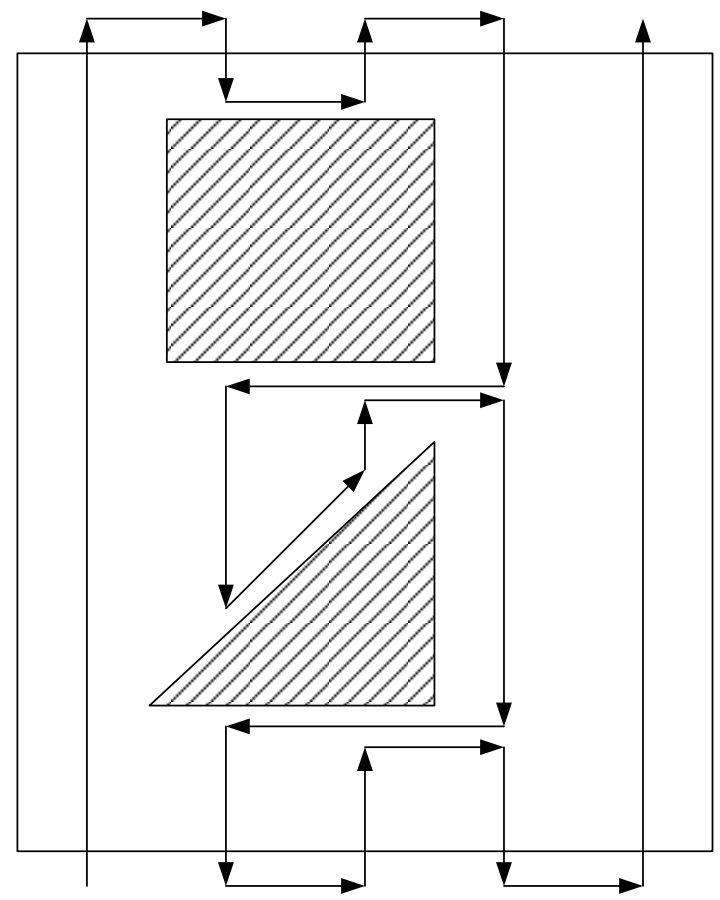

Figure 8. Snake-like Scheme of the Mobile Beacon Node in Obstacle Surrounding 


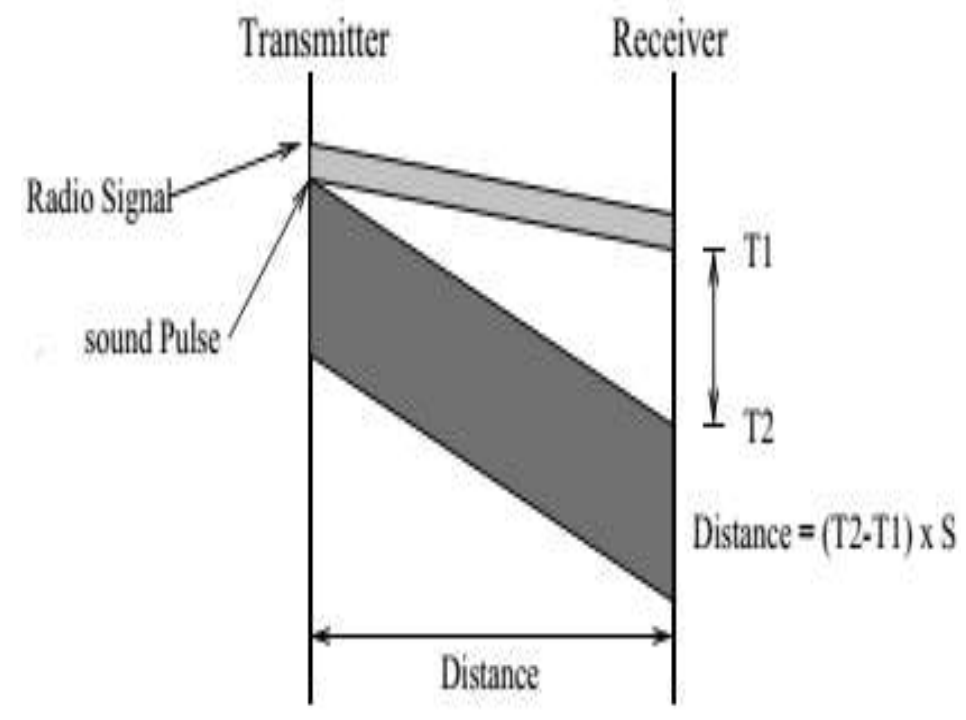

Figure 9. Distance Measurement using Sound and Radio Signals

The time difference $\left(T_{2}-T_{1}\right)$ is measured between two simultaneously received radio and sound pulse signals at sensor node in Figure 9. The speeds of radio and sound is supposed to $c_{1}$ and $c_{2}$ respectively. Then the distance

$$
d=\left(T_{2}-T_{1}\right) S=\left(T_{2}-T_{1}\right) \frac{c_{1} c_{2}}{c_{1}-c_{2}}
$$

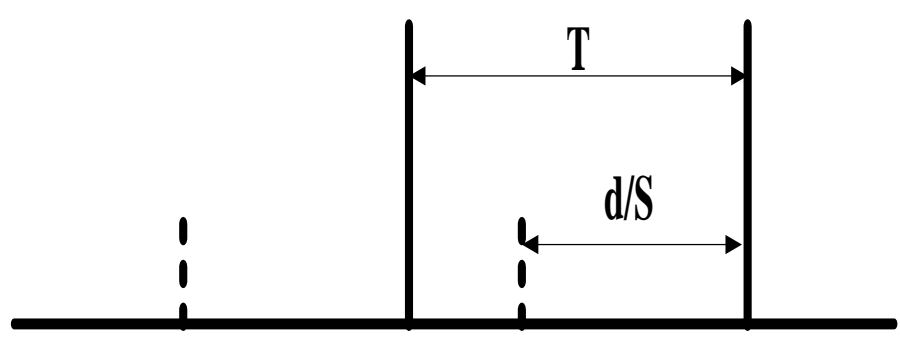

$$
\text { sound2 radio2 sound1 radiol }
$$

Figure 10. Intermessage Delay Limitation

As is displayed in Figure 10, to avoid ambiguous distance, the intermessage delay $T$ must satisfy

there into

$$
T>d / S
$$

$$
d \leq R
$$

So

$$
T>\frac{R\left(c_{1}-c_{2}\right)}{c_{1} c_{2}}
$$

Let $T=\frac{R}{c_{2}}$, As is displayed in Figure 11, when $i d=1$, the sensor node coordinate is 


$$
\left(x_{s}, y_{s}\right)=\underset{\left(x_{a}+d, y_{a}\right)}{\arg \min }\left(T_{2}-T_{1}\right)
$$

Accordingly, when $i d=2$, the sensor node coordinate is

$$
\left(x_{s}, y_{s}\right)=\underset{\left(x_{a}-d, y_{a}\right)}{\arg \min }\left(T_{2}-T_{1}\right)
$$

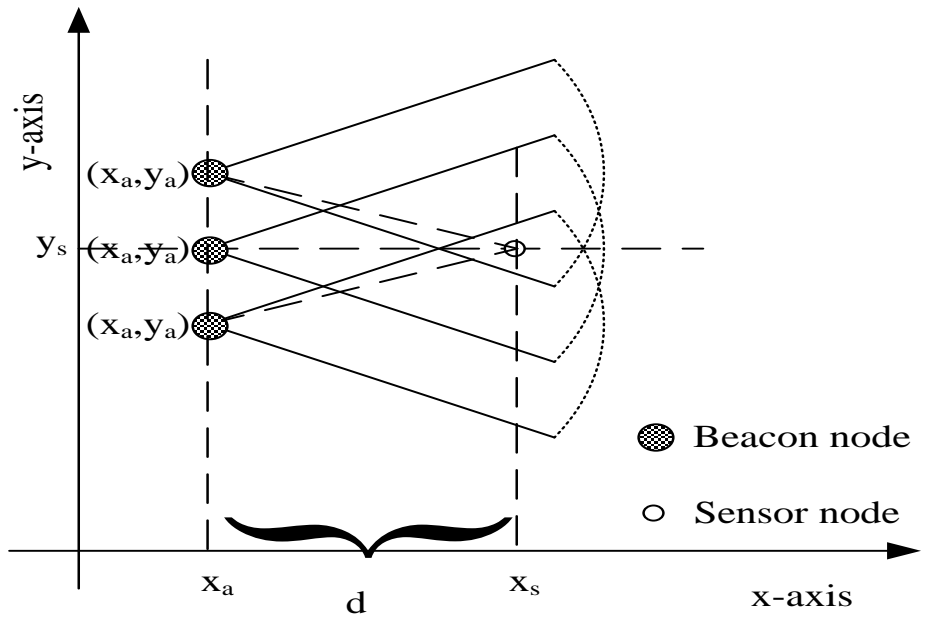

Figure 11. An Example of Location Estimation

\section{Localization Error Analysis}

The beacon node is supposed to travel through the WSN with constant velocity $v \mathrm{~m} / \mathrm{s}$. The distance between successive beacon message is obviously $v T$ which is defined as the beacon distance.

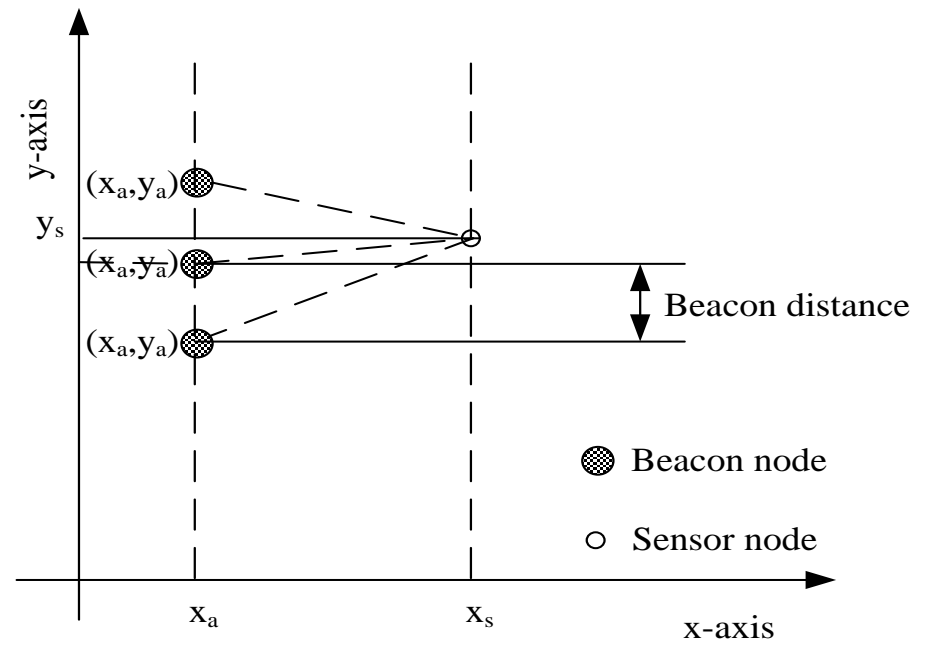

Figure 12. Localization Error Analysis of y-coordinate

As is displayed in Figure 12, we have

$$
e_{y_{-} \text {beacon }} \sim U\left(-\frac{v T}{2}, \frac{v T}{2}\right)
$$

Taking the measurement error of the distance $d$ into consideration[26], It is assumed that 


$$
e_{x_{-} \text {beacon }} \sim U(-0.1 d, 0.1 d)
$$

Clearly, the accuracy of the DAS localization scheme is dependent on the GPS error, beacon distance. Suppose that GPS error satisfies

$$
\left(e_{x_{-} g p s}, e_{y_{-} g p s}\right) \sim N\left(0,0, \sigma_{1}^{2}, \sigma_{2}^{2}, \rho\right)
$$

So

$$
\begin{gathered}
e_{y}=e_{y_{-} g p s}+e_{y_{-} \text {beacon }} \\
e_{x}=e_{x_{-} g p s}+e_{x_{-} \text {beacon }} \\
E_{D}\left(e_{x}^{2}\right)=E_{D}\left(\left(e_{x_{-} g p s}+e_{x_{-} \text {beacon }}\right)^{2}\right) \\
=E_{D}\left(e_{x_{-} g p s}^{2}\right)+E_{D}\left(e_{x_{-} \text {beacon }}^{2}\right) \\
=\sigma_{1}^{2}+\frac{(0.2 D)^{2}}{12}(0 \leq D \leq R) \\
E_{D}\left(e_{y}^{2}\right)=E_{D}\left(\left(e_{y_{-} g p s}+e_{y_{-} \text {beacon }}\right)^{2}\right) \\
=E_{D}\left(e_{y_{-} g p s}^{2}\right)+E_{D}\left(e_{y_{-} \text {beacon }}^{2}\right) \\
=\sigma_{2}^{2}+\frac{(v T)^{2}}{12} \\
E\left(e^{2}\right)=\frac{1}{D} \int_{0}^{R} E_{D}\left(e_{x}^{2}+e_{y}^{2}\right) d D \\
=\frac{1}{D} \int_{0}^{R} E_{D}\left(e_{x}^{2}\right)+E_{D}\left(e_{y}^{2}\right) d D \\
=\sigma_{1}^{2}+\sigma_{2}^{2}+\frac{0.01 R^{3}}{9}+\frac{(v T)^{2}}{12} \\
+\sigma_{2}^{2}+\frac{0.01 R^{3}}{9}+\frac{(v R)^{2}}{12 c_{2}^{2}}
\end{gathered}
$$

\section{Localization Property in Obstacle Surroundings}

Obstacle surroundings couldn't be avoided in the most of wireless sensor network applications. In the localization scheme, the good property of localization is displayed in Figure 13. when the sensor node locates besides obstacle and receives two beacon message (1) and (2). (2) is received through reflection propagation of which the distance is more far than the distance of (1). According to formula (1) (2), the larger localization error is avoided. 


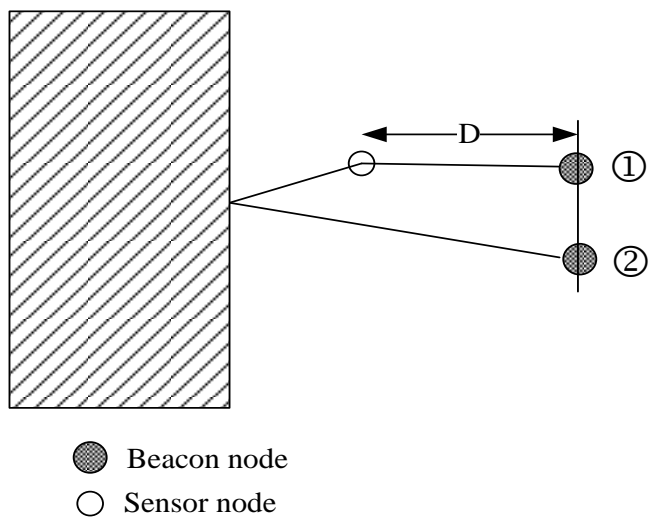

(a) Rectangle Obstacle

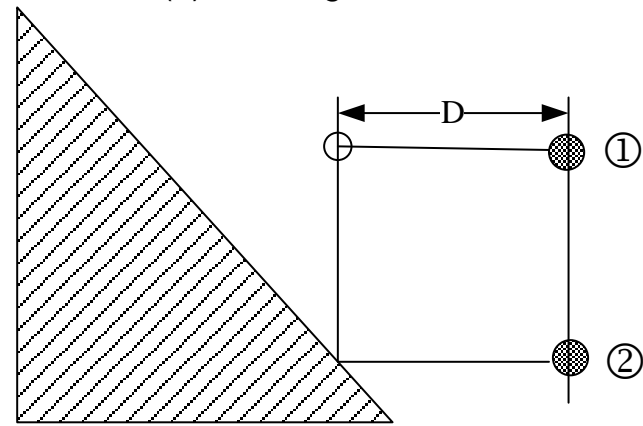

Beacon node

Sensor node

(b) Triangle Obstacle

Figure 13. Localization in Obstacle Surroundings

\section{Simulation}

In this section, the localization error is discussed through various simulation conditions. The scope of sensor field is assumed to $500 \times 500 \mathrm{~m} 2$ and 1000 sensor nodes are randomly deployed in it. The mobile beacon node is fitted with a GPS receiver, two directional antennas, two directional sound sources and a digital compass. It travels in straight lines through the WSN. Each sensor node is fitted with omni-directional antenna transceiver and a microphone. The remained simulation parameters are given in Table 1. the default values are displayed within parentheses. To guarantee the representative and reliability of the computer simulation results, a total of 100 simulations were implemented for each series of simulation conditions, with various original random sensor node deployment.

Table 1. Simulation Parameters

\begin{tabular}{l|l}
\hline Parameter & Value \\
\hline Distance between two adjacent line $2 R(\mathrm{~m})$ & $10,(25), 40$ \\
\hline Moving speed $v(\mathrm{~m} / \mathrm{sec})$ & $10,(20), 30$ \\
\hline The speed of sound $c_{2}(\mathrm{~m} / \mathrm{sec})$ & 340 \\
\hline$\sigma_{1}=\sigma_{2}(\mathrm{~m})$ & 1 \\
\hline Beam width(degrees) & 5 \\
\hline
\end{tabular}




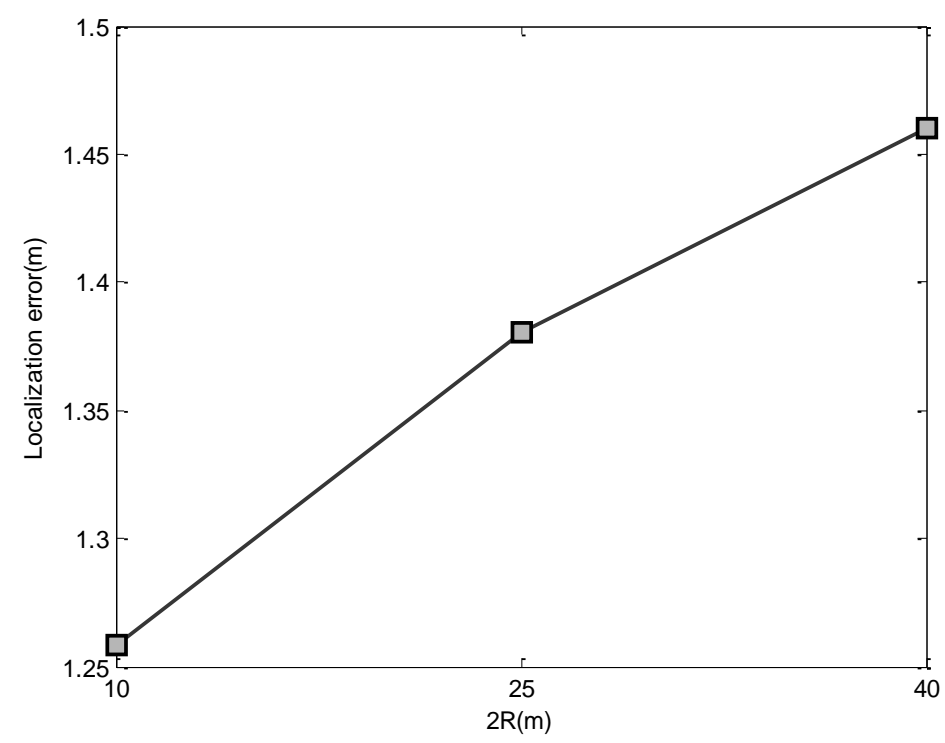

Figure 14. Localization error Versus Distance between Two Adjacent Line

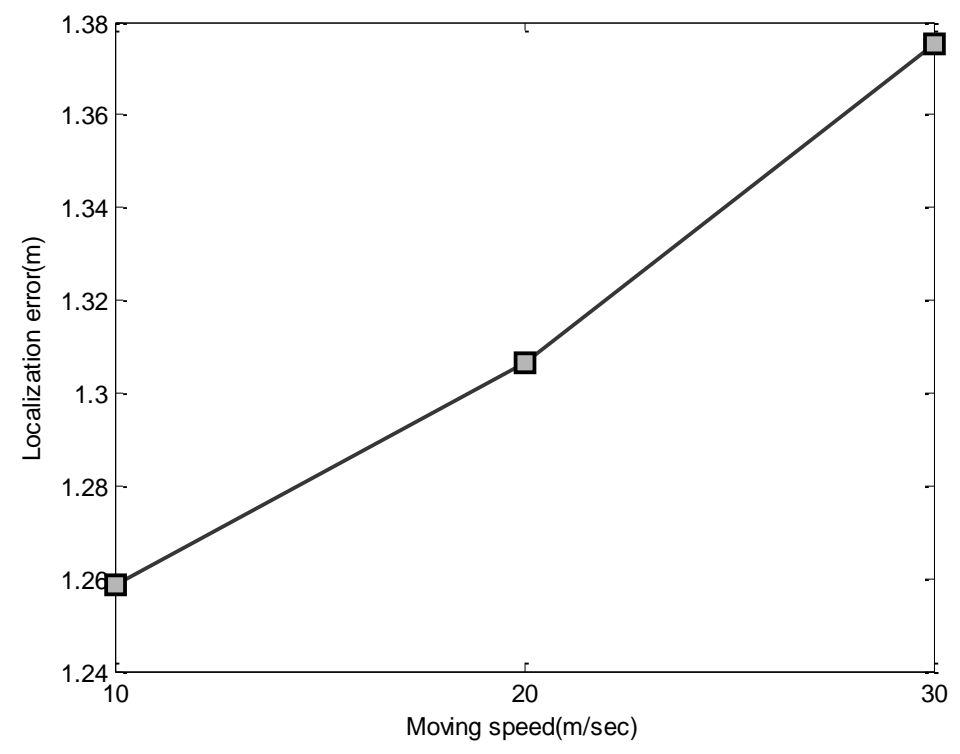

Figure 15. Localization Error Versus Moving Speed

The performance of the DAS scheme was evaluated for various distances of two adjacent line and various traveling speeds as displayed in Figure 14 and Figure 15. It was found to be consistent with analysis above.

To explain the DAS scheme's immune to obstacle surroundings, we consider that a rectangle obstacle and a triangle obstacle is in the WSN surroundings as displayed in Figure 8. Confronting obstacles, the mobile beacon node adopts snake-like scheme. As is displayed in Figure 16, the obstacles have little effect on the performance of the DAS scheme. 


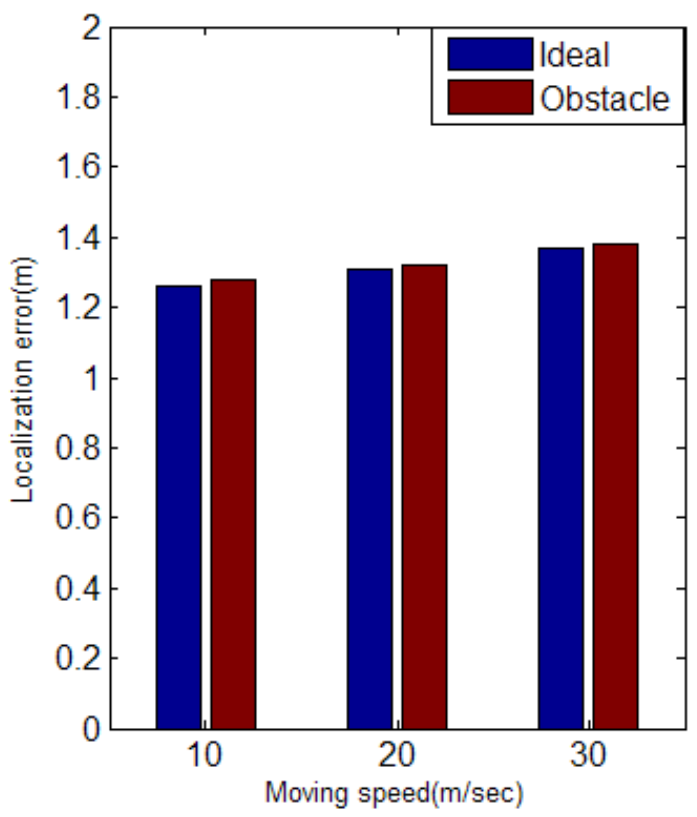

Figure 16. Localization Error Versus Surroundings

\section{Conclusions}

In this paper, we address shortcomings of existing sensor localization schemes. Then, the idea of multi-modal schemes using directional antenna and sound source is explored to compute locations of sensors in WSN. In the proposed approach, designated as DAS, mobile beacon nodes are fitted with two directional antennas and two directional sound sources, as they travel through the WSN. Sensor node doesn't need extra hardware requirements in the DAS scheme. Through error analysis and computer simulation, it has been shown that DAS is robust toward both parameters of WSN and the mobile beacon node, yet immune to the presence of obstacles in the sensing surroundings.

For next work, we intend to implement analysis of the communication costs during the manipulation of our schemes. The experiments associated with energy consumption and message complexity will be done. We also intend to research localization schemes about mobile sensor nodes.

\section{References}

[1] I. F. Akyildiz, W. Su, Y. Sankarasubramaniam and E. Cayirci, "A survey on sensor networks", IEEE Commun. Mag., vol. 40, no. 8, (2002), pp. 102-114.

[2] A. Yilmaz, O. Javed and M. Shah, "Object tracking: A survey", ACM Journal of Computing Surveys, vol. 38, (2006), pp. 13-57.

[3] C. F. Huang and Y. C. Tseng, "The coverage problem in a wireless sensor network", Mobile Netw. Appl., vol. 10, no. 4, (2005), pp. 519-528.

[4] I. Stojmenovic, "Position-based routing in ad hoc networks", IEEE Commun. Mag., vol. 40, no. 7, (2002), pp. 128-134.

[5] B. Hofmann-Wellenhof, H. Lichtenegger and J. Collins, "Global Positioning System: Theory and Practice", Berlin, Germany: Springer-Verlag, (1997).

[6] P. Bahl and V. N. Padmanabhan, "RADAR: An in-building RF-based user location and tracking system", Proc. IEEE Joint Conf. IEEE Comput. Commun. Societies (INFOCOM), Tel Aviv, Israel, (2000) March 26-30.

[7] N. Bulusu, J. Heidemann and D. Estrin, "GPS- less low-cost outdoor localization for very small devices", IEEE Pers. Commun., vol. 7, no. 5, (2000), pp. 28-34.

[8] J. Hightower, G. Borriello and R. Want, "SpotON: An indoor 3D location sensing technology based on RF signal strength”, University of Washington, Seattle, WA, Tech. Rep. UW CSE 00-02-02, (2000). 
[9] A. Savvides, C. C. Han and M. B. Srivastava, "Dynamic fine-grained localization in ad-hoc networks of sensors", Proc. ACM Int. Conf. Mobile Comput. Networking (MOBICOM), Rome, Italy, (2001) July 16-21.

[10] A. Savvides, H. Park and M. Srivastava, "The bits and flops of the n-hop multilateration primitive for node localization problems", Proc. 1st ACM Int. Workshop Wireless Sensor Networks Applicat.-WSNA 02, Atlanta, GA, (2002) September 28.

[11] C. Savarese, J. Rabaey and K. Langendoen, "Robust positioning algorithms for distributed ad-hoc wireless sensor networks", Proceedings of the General Track of the annual conference on USENIX Annual Technical Conference (ATEC '02), USENIX Association Berkeley, CA, USA, (2002) June 1015.

[12] T. He, C. Huang, B. M. Blum, J. A. Stankovic and T. Abdelzaher, "Range-free localization schemes for large scale sensor networks", Proc. ACMInt. Conf. Mobile Comput. Networking (MOBICOM), San Diego, California, USA, (2003) September 14-19.

[13] D. Niculescu and B. Nath, "DV based positioning in ad hoc networks", Kluwer J. Telecommun. Syst., vol. 22, no. 1, (2003), pp. 267-280.

[14] N. Patwari, A. O. Hero, M. Perkins, N. S. Correal and R. J. ODea, "Relative location estimation in wireless sensor networks”, IEEE Trans. Signal Process., vol. 51, no. 8, (2003), pp. 2137-2148.

[15] K. K. Chintalapudi, A. Dhariwal, R. Govindan and G. Sukhatme, "Ad-hoc localization using ranging and sectoring", Proc. IEEE Joint Conf. IEEE Comput. Commun. Societies (INFOCOM), hong kong, china, (2004) March 7-11.

[16] N. Malhotra, M. Krasniewski, C. L. Yang, S. Bagchi and W. Chappell, "Location estimation in ad-hoc networks with directional antenna", Proc. IEEE Int. Conf. Distrib. Comput. Syst (ICDCS), Columbus, Ohio, United States, (2005) July 6-10.

[17] N. Patwari, J. N. Ash, S. Kyperountas, A. O. Hero, R. L. Moses and N. S. Correal, "Locating the nodes: Cooperative localization in wireless sensor networks", IEEE Signal. Process. Mag., vol. 22, no. 4, (2005), pp. 54-69.

[18] V. Vivekanandan and V. W. S. Wong, "Concentric anchor beacon localization algorithm for wireless sensor networks”, IEEE Trans. Veh. Technol., vol. 56, no. 5, (2007), pp. 2733-2744.

[19] L. Doherty, K. Pister and L. Ghaoui, "Convex position estimation in wireless sensor networks", Proceedings of IEEE INFOCOM'01, Alaska, USA, vol. 3, (2001) April 22-26.

[20] K. F. Ssu, C. H. Ou and H. C. Jiau, "Localization with mobile anchor points in wireless sensor networks", IEEE Trans, Veh. Technol., vol. 54, no. 3, (2005), pp. 1187-1197.

[21] B. Xiao, H. Chen and S. Zhou, "Distributed localization using a moving beacon in wireless sensor networks", IEEE Trans. Parallel and Distributed Systems, vol. 19, no. 5, (2008), pp. 587-600.

[22] S. Lee, E. Kim, C. Kim and K. Kim, "Localization with a mobile beacon based on geometric constraints in wireless sensor networks", IEEE Trans. Wireless Commun., vol. 8, no. 12, (2009), pp. 5801-5805.

[23] B. Zhang and F. Yu, "LSWD: Localization Scheme for Wireless Sensor Networks using Directional Antenna”, IEEE Transactions on Consumer Electronics, vol. 56, no. 4, (2010), pp. 2208-2216.

[24] C. H. Ou, "A Localization Scheme for Wireless Sensor Networks Using Mobile Anchors With Directional Antennas", IEEE Sensors Journal, vol. 11, no. 7, (2011), pp. 1607-1616.

[25] A. Appelbaum and L. Kaplan, "Sidelobe suppression considerations in the design of an electronically steered IFF antenna", IEEE Trans. Antennas Propagat., vol. AP-24, no. 4, (1976), pp. 425-432.

[26] C. Dean Whitehouse, "The Design of Calamari: an Ad-hoc Localization System for sensing Networks", Master of Science in Electrical Engineering and Computer Science, (2002).

[27] D. Koutsonikolas, M. Saumitra, Y. Das and C. Hu, "Path planning of mobile landmarks for localization in wireless sensor networks", Computer Communications, vol. 30, (2007), pp. 2577-2592.

[28] S. M. Mazinani and F. Farnia, "Localization in Wireless Sensor Network Using a Mobile Anchor in Obstacle Environment", International Journal of Computer and Communication Engineering, vol. 2, no. 4, (2013), pp. 438-441.

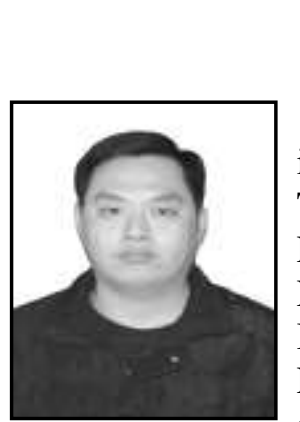

\section{Authors}

Qinli An, was born in 1975. and received the bachelor degree in Applied Maths from National University of Defense Technology in China, master degree in Applied Maths from Air Force Engineering University in China and a Ph.D. student from Northwestern Polytechnical University in Communication and Information Engineering. $\mathrm{He}$ is a lecturer in Air Force Engineering University. His current research interests are mobile ad hoc networks with directional antenna and wireless sensor networks. E-mail: anqinli@126.com 